\title{
Novel Conjugated Polymer Containing Anthracene Backbone: Addition Polymers of 1,4-Benzenedithiol with 9,10- Diethynylanthracene and Its Conductivity
}

\author{
Eiichi KOBAYASHI, ${ }^{*}$ Jian JIANG, and Junji FURUKAWA \\ Department of Industrial Chemistry, Faculty of Science and Technology, \\ Science University of Tokyo, Noda, Chiba 278, Japan
}

(Received September 28, 1989)

\begin{abstract}
The addition polymerization of 1,4-benzenedithiol to 9,10-diethynylanthracene, which was freshly prepared before use, was carried out in a THF and benzene solution at preper temperature under nitrogen atmosphere by various initiation methods such as UV irradiation, AIBN and BPO. The yield of polymer was affected seriously by the initiation mode. Both the yield and the molecular weight are higher for the polymer prepared by UV irradiation than that prepared by means of AIBN or BPO. By UV irradiation, the yield of the polymer has reached about $60 \%$, and the molecular weight of the polymer $\left(\bar{M}_{n}\right)$ obtained in THF was about 80000 , which is one order higher than that of the polymer obtained in benzene. The electrical conductivity of the polymer is in the order of $10^{-13} \mathrm{~S} \mathrm{~cm}^{-1}$ without doping, but increased up to $10^{-6} \mathrm{~S} \mathrm{~cm}^{-1}$ on $\mathrm{I}_{2}$ doping. The diffuse reflectance spectra of the polymer indicate that the polymer has relatively lower band gap (about $1.77 \mathrm{eV}$ ), and at $1.46 \mathrm{eV}$ and $0.86 \mathrm{eV}$ small peaks appear, which may concern with a polaron or bipolaron in the polymer backbone.

KEY WORDS Addition Polymerization / Conjugated Polymer / 1,4Benzenedithiol / 9,10-Diethynylanthracene / $\mathrm{I}_{2}$ Doping / Electrical Conductivity / Diffuse Reflectance Spectra / TG / DTA /
\end{abstract}

It was known previously that the addition polymerization of 1,4-benzenedithiol to 1,4diethynylbenzene in solid state gave a highly oriented crystalline polymer film. The polymer has the $10^{-2} \mathrm{~S} \mathrm{~cm}^{-1}$ electrical conductivity on $\mathrm{I}_{2}$ doping, and its crystallinity and the $c i s$ and trans contents of vinylene units could be reversibly changed by the UV irradiation with special wavelengths. ${ }^{1-4}$ There are also synthesized novel conjugated polymers of $4,4^{\prime}$ diethynylbiphenyl and 4,4'-biphenyldithiol, and heterocyclic aromatic polymers which contain a sulfur or nitrogen atom in the polymer backbone. ${ }^{5-8}$ These polymers have been attracting a great deal of attention because of their unusual electronic and optical properties.

In other way, Nuyken and Siebzehnrübl ${ }^{9}$ have synthesized sulfur containing polymers by using the radical polymerization of 1,3benzenedithiol to 1,5-hexadiyne, which are soluble in the common solvents such as benzene, toluene and chloroform in contrast to our polymers $^{1,2}$ mentioned above.

In this paper the authors have tried to synthesize a new conjugated polymer containing the polycyclic aromatic monomer unit such as 9,10-anthracenedithiol or 9,10-diethynylanthracene instead of benzene derivatives. Firstly, 9,10-diethynylanthracene (DEA) was synthesized and polymerized with 1,4-benzenedithiol (BDT) to obtain a new conjugated polymer [1], and its electrical properties were also investigated.

* To whom all correspondence should be addressed. 


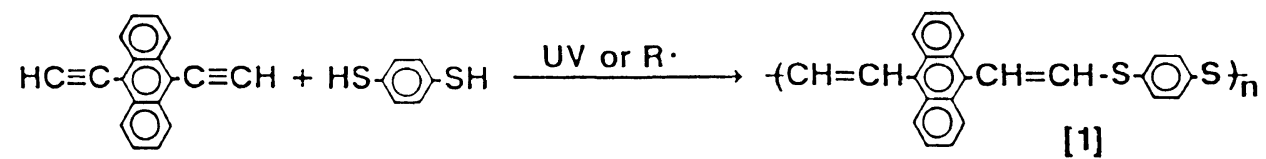

\section{EXPERIMENTAL}

Preparation of 9,10-Bis(trimethylsilylethynyl)anthracene

$12 \mathrm{~g}(0.035 \mathrm{~mol})$ of 9,10-dibromoanthracene ${ }^{10}$ recrystallized from a $\mathrm{CCl}_{4}$ solution was charged in one liter of four necked flask fitted with effective condenser and stirrer. Then, $500 \mathrm{ml}$ of diethyl amine (Wako Pure Chemical Industries), $14 \mathrm{~g}(0.14 \mathrm{~mol})$ of trimethylsilylacetylene (Shinetsu Chem. Co.), $1 \mathrm{~g}$ $(0.0014 \mathrm{~mol})$ of bis(triphenylphosphine)paradiumdichloride $^{11}$ and $0.55 \mathrm{~g}(0.0028 \mathrm{~mol})$ of copper iodide (Wako Pure Chemical Industries) were added into the flask under nitrogen atmosphere. The reaction was carried out under nitrogen atmosphere at the reflux temperature for $4 \mathrm{~h}$. The reaction mixture was filtered to exclude the solid residues containing metal compounds. The filtrate was condensed into solid masses by evaporation, which was dissolved in benzene and purified with an activated alumina column. The hexane-benzene $1: 1$ solution was used as an eluent. After evaporating the solution, 9,10-bis(trimethylsilylethynyl)anthracene was obtained as yellow powder. It was further purified by recrystallization in benzene. Yield: $12.7 \mathrm{~g}(95 \%)$. mp: $228-229$ C. ${ }^{1} \mathrm{H}$ NMR: (d-chloroform, TMS as standard), $\delta$ in ppm: $0.4\left(\mathrm{~s},-\mathrm{CH}_{3}, 9 \mathrm{H}\right)$; 7.33 (q, anthracene, $2 \mathrm{H}$ ); 8.33 (q, anthracene, 2H). IR (KBr): $2150 \mathrm{~cm}^{-1}(\mathrm{w}, \delta(-\mathrm{C} \equiv \mathrm{C}-))$; $1380 \mathrm{~cm}^{-1}$ (s, $\left.\delta\left(-\mathrm{CH}_{3}\right)\right) ; 1250,860 \mathrm{~cm}^{-1}$ (vs, $v$ $\left.\left(-\mathrm{SiMe}_{3}\right)\right) ; 770 \mathrm{~cm}^{-1}$ (s, $\delta$ (substituted anthracene ring)). Mass: $\mathbf{M}^{+}, 370, \mathbf{M}+1^{+}, 371$.

\section{9,10-Diethynylanthracene}

$2 \mathrm{~g}(0.0054 \mathrm{~mol})$ of 9,10-bis(trimethylsilylethynyl)anthracene was added under nitrogen atmosphere into one liter of four necked flask fitted with stirrer and thermometer, and then, $300 \mathrm{ml}$ of fresh diethyl ether, $200 \mathrm{ml}$ of methanol and $100 \mathrm{ml}$ of $1 \mathrm{~N} \mathrm{NaOH}$ aqueous solution were charged. After hydrolysis at room temperature for $2 \mathrm{~h}, 100 \mathrm{ml}$ of diethyl ether was additionally added in the above reaction mixture, then, it was washed with water until $\mathrm{pH}$ approximately equals to 7 . The diethyl ether solution was dried over anhydrous $\mathrm{MgSO}_{4}$ for about $4 \mathrm{~h}$. After evaporation of diethyl ether the residue was recrystallized in hexane. $1.02 \mathrm{~g}$ (yield $83 \%$ ) of 9,10 diethynylanthracene was obtained as yellow crystal. Its melting point could not be determined because it changed to black at $85^{\circ} \mathrm{C}$ and did not melt below $300^{\circ} \mathrm{C}$. ${ }^{1} \mathrm{H}$ NMR: $\left(\mathrm{CCl}_{4}, \mathrm{TMS}\right.$ as standard), $\delta$ in ppm: 3.82 (s, $(\equiv \mathrm{C}-\mathrm{H}), \mathrm{H}) ; 7.33$ (q, anthracene, $2 \mathrm{H}) ; 8.33$ (q, anthracene, $2 \mathrm{H})$. IR $(\mathrm{KBr}): 3300 \mathrm{~cm}^{-1}(\mathrm{~s}, v$ $(\equiv \mathrm{C}-\mathrm{H})) ; 2100 \mathrm{~cm}^{-1}(\mathrm{w}, \delta(-\mathrm{C} \equiv \mathrm{C}-)) ; 760$ $\mathrm{cm}^{-1}$ (vs, $\delta$, substituted anthracene ring)). Mass: $\mathrm{M}^{+}, 226, \mathrm{M}+1^{+}, 227$.

The synthetic route of 9,10-diethynylanthracene is illustrated in Scheme 1.

\section{1,4-Benzenedithiol}

1,4-Benzenedithiol was prepared according to the reference ${ }^{2}$ and newly sublimated at $70^{\circ} \mathrm{C}$ under $0.5 \mathrm{mmHg}$ before use; $\mathrm{mp}: 96-97^{\circ} \mathrm{C}$ (mp: $97 \mathrm{C}^{12}$ ).

\section{Polymerization}

The polymerization was carried out in a
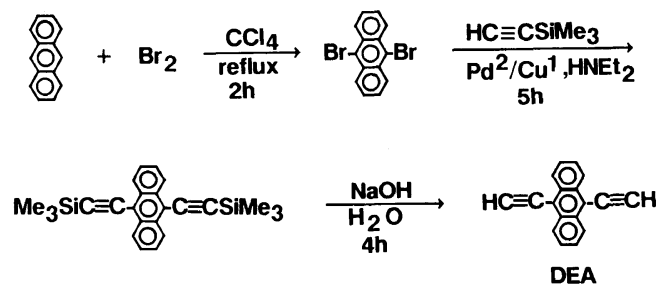

Scheme 1. Synthetic route of 9,10-diethynylanthracene. 
pyrex tube in a THF or benzene solution by UV irradiation, using high pressure mercury lamp 450W (Ushio Co.) under nitrogen atmosphere. The precipitated insoluble polymer was recovered by a centrifugation, washed with THF or benzene and dried in vacuo at room temperature (the insoluble part). When methanol was poured into the residual THF or benzene solution, the polymer was precipitated. By a centrifugation, the soluble polymer was recovered. After washing out the residual monomers with methanol, it was dried in vacuo at room temperature for one night (the soluble part).

\section{Characterization}

For the characterization of monomers and the polymers the following instruments were used: ${ }^{1} \mathrm{H}$ NMR: JEOL PMX-60si; IR: Hitachi 260-50; Diffuse reflectance spectra: Hitachi UV-330; DTA.TG: SINKU-RIKO TGD-3000 Differential thermal microbalance connected with TA-1500 thermal analyzer. Mass spectra: JMS D-300; The content of sulfur in the polymers was determined by means of Schoeniger's method. The molecular weight of the polymers was measured by the determination of the end groups of polymers which is so called copper acetylide method. ${ }^{1}$

The electroconductivity was measured by following two-terminal method with an Electro Multi Meter (Takeda Riken Co.). $\mathrm{I}_{2}$ doping was carried out by exposing the polymer powder to iodine vapor at $40^{\circ} \mathrm{C}$ under $0.5 \mathrm{mmHg}$. The doped polymer powder was pressed to a pellet (diameter $10 \mathrm{~mm}$, thickness: $0.5-1 \mathrm{~mm}$ ). A platinum lead wire was connected to the pellet by Electrodag 502 (Furuuchi Kagaku Co.).

\section{RESULTS AND DISCUSSION}

\section{Addition Polymerization}

The addition polymerization of 1,4-benzenedithiol to 1,4-diethynylbenzene proceeds readily by UV irradiation or conventional initiators such as $2,2^{\prime}$-azobisisobutyronitrile, benzoyl peroxide and a trace amount of oxygen remaining in the polymerization tube. ${ }^{1}$ This sort of the addition polymerization progresses on a kind of radical formed by UV irradiation, radical initiator or oxygen.

Table I shows the effects of the initiation mode and the reaction time on the polymerization of BDT to DEA in a THF solution. As shown in Table I, the UV irradiation and radical initiator are more effective to the polymer yield and the molecular weight than without initiators. The yield and the number average molecular weight $\left(\bar{M}_{n}\right)$ are higher by

Table I. Effect of initiation mode and reaction time on the polymerization of BDT to DEA in THF at $50^{\circ} \mathrm{C}$ under nitrogen atmosphere ${ }^{\mathrm{a}}$

\begin{tabular}{|c|c|c|c|c|c|}
\hline \multirow{2}{*}{ No. } & \multirow{2}{*}{$\begin{array}{l}\text { Initiation } \\
\text { mode }\end{array}$} & \multirow{2}{*}{$\frac{\text { Reaction time }}{\min }$} & \multicolumn{3}{|c|}{ Insoluble polymer } \\
\hline & & & Yield $/ \%$ & $\bar{M}_{n} \times 10^{-3 \mathrm{~b}}$ & $\mathrm{~S} / \mathrm{wt} \% \mathrm{c}$ \\
\hline 1 & UV & 10 & 16 & 67 & 17.2 \\
\hline 2 & UV & 20 & 35 & 85 & 17.3 \\
\hline 3 & UV & 120 & 55 & 89 & 17.2 \\
\hline 4 & AIBN $^{d}$ & 20 & 48 & 67 & 17.7 \\
\hline 5 & None ${ }^{e}$ & 20 & 27 & 58 & 17.1 \\
\hline
\end{tabular}

a $[\mathrm{BDT}]=[\mathrm{DEA}]=8.5 \times 10^{-2} \mathrm{moll}^{-1}$.

b Copper acetylide method.

c Schoeniger's method, $\mathrm{S} w \mathrm{w} \%$ of $1: 1$ polymer is 17.4 .

d $[$ AIBN $]=1.64 \times 10^{-3} \mathrm{moll}^{-1}$.

e In dark. 
UV irradiation than by others. The molecular weight of polymers increased with the polymerization time. These results indicate that the addition polymerization proceeds with the radical and the behavior of the molecular weight indicates a stepwise polymerization. The molecular weight would be controlled by the polymerization time. The expected reaction scheme is as follows (Scheme 2).

The polymerization in a benzene solution is shown in Table II. In the benzene solution, the UV irradiation and radical initiators such as AIBN and BPO have almost the same effect on the polymerization. It is noteworthy that the molecular weight of the polymers obtained in benzene was much lower than that of the polymers obtained in THF. The solubility of polymers in THF may be a little better than in

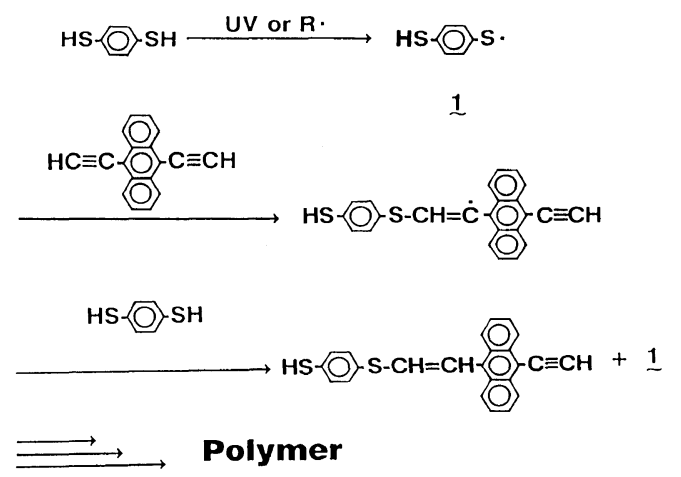

Scheme 2. The mechanism of addition polymerization. benzene. The addition reaction of polymer ends with monomers is conveniently proceeded in the THF solution. There is not so much difference in the molecular weight of the insoluble and the soluble polymers. The post polymerization of the ethynyl and thiol end groups of the soluble polymers may occur during the preservation of polymers in a desiccator.

The yield of the insoluble polymer increased at first with the polymerization time, then decreased a little as shown in Figure 1. It seems that UV photolysis of the resulting insoluble polymer would happen, so that the insoluble

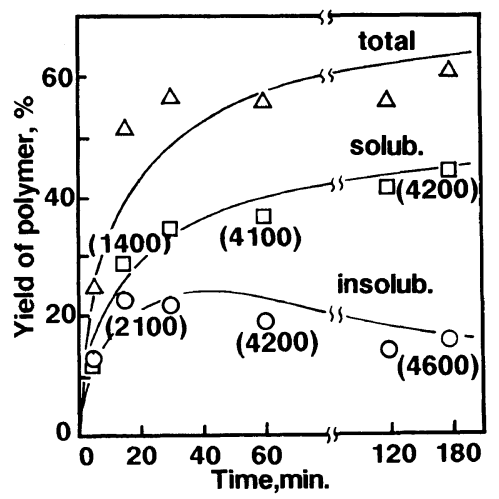

Figure 1. Relationship between reaction time and the yield of DEA-BDT polymer obtained by UV irradiation in benzene at $60^{\circ} \mathrm{C}$ under nitrogen atmosphere. [DEA] $=$ $[\mathrm{BDT}]=7.3 \times 10^{-2} \mathrm{moll}^{-1}$. High pressure mercury lamp of $450 \mathrm{~W}$. ( ) means $\bar{M}_{n}$.

Table II. Effect of initiation mode on the polymerization of BDT to DEA in benzene at $60^{\circ} \mathrm{C}$ for $60 \mathrm{~min}$ under nitrogen atmosphere ${ }^{\mathrm{a}}$

\begin{tabular}{|c|c|c|c|c|c|c|}
\hline \multirow{2}{*}{ No. } & \multirow{2}{*}{$\begin{array}{l}\text { Initiation } \\
\text { mode }\end{array}$} & \multicolumn{2}{|c|}{ Yield $/ \%$} & \multicolumn{2}{|c|}{$\bar{M}_{n}^{\mathrm{b}}$} & \multirow{2}{*}{$\frac{\mathrm{S} / \mathrm{wt} \%}{\text { Insol. }}$} \\
\hline & & Insol. & Sol. & Insol. & Sol. & \\
\hline 1 & UV & 19 & 37 & 4200 & 4100 & 17.8 \\
\hline 2 & $\mathrm{AIBN}^{\mathrm{d}}$ & 27 & 6 & 4000 & 3600 & 17.8 \\
\hline 3 & $\mathrm{BPO}^{\mathrm{d}}$ & 28 & 8 & 3700 & 3100 & 18.0 \\
\hline 4 & None $e^{e}$ & 27 & 6 & 3700 & 3100 & 18.2 \\
\hline
\end{tabular}

a $[\mathrm{BDT}]=[\mathrm{DEA}]=7.3 \times 10^{-2} \mathrm{moll}^{-1}$.

b Copper acetylide method.

c Schoeniger's method, $\mathrm{S} w \mathrm{t} \%$ of $1: 1$ polymer is 17.4 .

${ }^{d}[\mathrm{AIBN}]=[\mathrm{BPO}]=1.7 \times 10^{-3} \mathrm{moll}^{-1}$.

e In dark. 


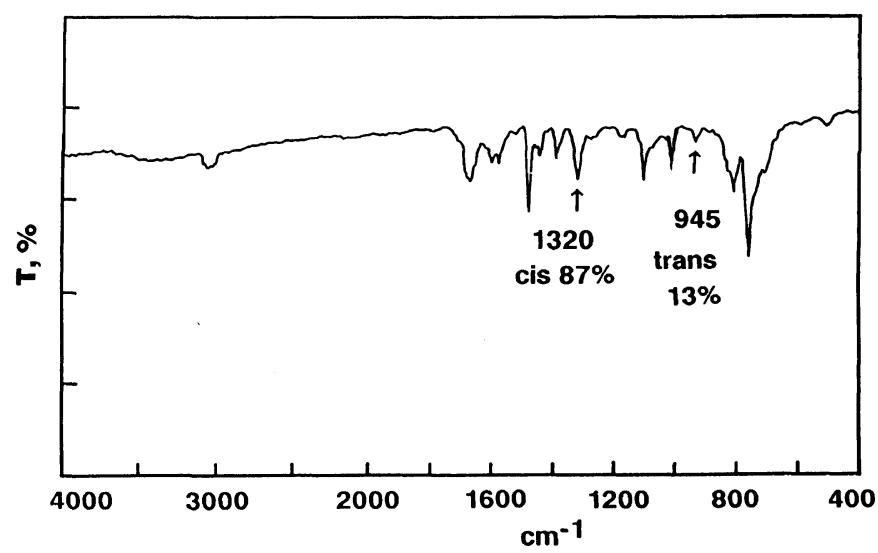

Figure 2. IR spectrum of DEA-BDT insoluble polymer (Table I, No. 3) by KBr method.

polymer decomposes to be soluble parts dissolving in the polymerization solution. When the obtained insoluble polymer was irradiated by UV light in benzene over again, a soluble low molecular weight fraction was obtained with about $20 \%$ yield, and the molecular weight of the insoluble polymer also decreased from 4200 to 3300 . From ${ }^{1} \mathrm{H}$ NMR determination, the soluble fraction has an irregular structure composed of anthracene, benzene, and aliphatic sulfide groups. After the initial stage of the polymerization, the monomer concentration decreases gradually and relatively a large amount of low molecular weight polymer could be produced, while the molecular weight of both the insoluble and the soluble polymers increased with the polymerization time, the same as the behavior in a THF solution.

The content of sulfur in polymers was almost equal to that calculated based on $1: 1$ polymer. Figure 2 is the IR spectrum of the insoluble polymer measured by $\mathrm{KBr}$ method. The absorption peak at $945 \mathrm{~cm}^{-1}$ is due to the trans vinylene vibration mode and at 1620 and $1320 \mathrm{~cm}^{-1}$ the cis vinylene absorption appears. The cis content is calculated to be $87 \%$. Besides at $3050 \mathrm{~cm}^{-1}$, disubstituted phenyl and disubstituted anthracene ring appear at $810 \mathrm{~cm}^{-1}$ and $750 \mathrm{~cm}^{-1}$, respectively. The absence of peaks between 400 and $500 \mathrm{~cm}^{-1}$ and

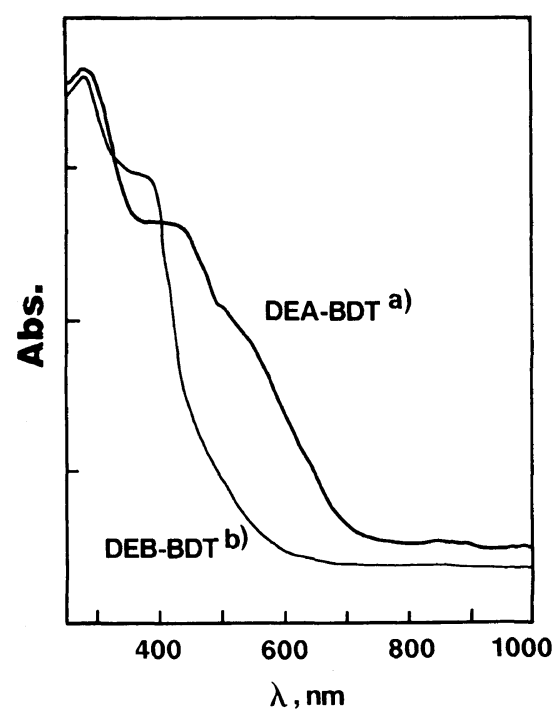

Figure 3. Diffuse reflectance spectra of undoped polymers: a) see Table I, No. 3; b) see ref 4 .

between 2800 and $2900 \mathrm{~cm}^{-1}$ indicates the absence of the $-\mathrm{S}-\mathrm{S}$ - disulfide bond and the branch formed by attacking of the thiol to the vinylene units in the polymer backbone. From the sulfur contents of the insoluble polymer and its IR spectrum, the obtained polymer was thought to be an alternating copolymer. The polymer end groups seem to be $-\mathrm{SH}$ or $-\mathrm{C} \equiv \mathrm{CH}$. However, in the IR spectrum the absorption peaks corresponding to these end groups were not found at 3300, 2500, and 
$2200 \mathrm{~cm}^{-1}$, because its molecular weight is high enough.

Figure 3 shows the diffuse reflectance spectra of the insoluble polymers. The polymer composed of 1,4-diethynylbenzene (DEB) and BDT has the maximum absorption at $380 \mathrm{~nm}$, but the polymer DEA-BDT [1] has the maximum absorption at $428 \mathrm{~nm}$ and tailing to $700 \mathrm{~nm}$. Surely, the conjugated $\pi$ system of the latter spreads over the polymer backbone much more than that of the former, because the structure of the latter has the vinylene anthracene unit and phenylene sulfide unit in the backbone, in which the $\pi$ electrons are much more convenient to delocalize along the polymer backbone.

The DTA and TG thermograms of the polymer [1] under air are shown in Figure 4. There is a small exothermic peak at $216^{\circ} \mathrm{C}$ in the DTA curve. On the other hand, in the DSC determined under nitrogen flow, the exothermic peak at $216^{\circ} \mathrm{C}$ was not observed. It seems that the peak at $216^{\circ} \mathrm{C}$ observed under air is likely to be a kind of chemical reaction, for example, a coupling reaction between anthracene rings or an addition reaction of oxygen to anthracene ring. But, a phase transition phenomenon is also possible way to explain the exothermic peak which was observed in the polymer of BDT with DEB. ${ }^{13}$ The polymer

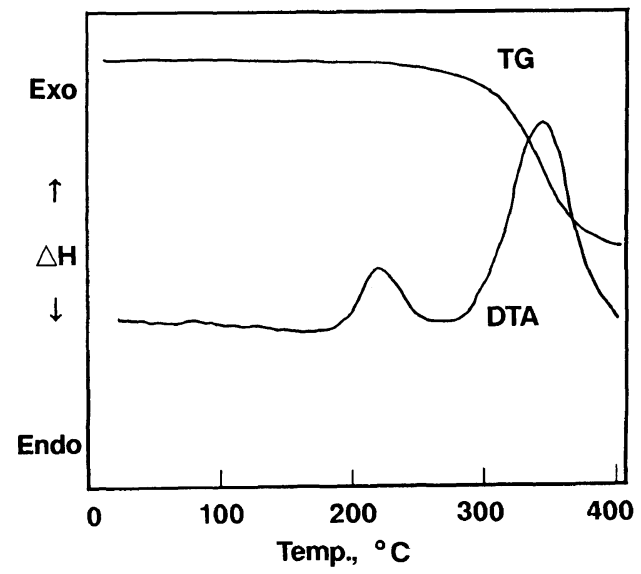

Figure 4. TG and DTA of the insoluble polymer (Table I, No. 3) under air: heating rate, $10^{\circ} \mathrm{C} \mathrm{min}^{-1}$. decomposed completely at about $350^{\circ} \mathrm{C}$. Its heat stability is worse than that of the polymer composed of 1,4-diethynylbenzene and BDT. The reason is that the anthracene ring is unstable against oxidation. The oxidation potential of anthracene, $1.09 \mathrm{~V}$ is much lower than that of benzene, $2.30 \mathrm{~V} .{ }^{14}$ As we know, the anthracene has a lower resonance energy of about $84 \mathrm{Kcal} / \mathrm{mol}$ than that of the expected, only slightly more than twice that of benzene. So it is unstable to many kinds of chemicals, especially to oxygen.

\section{Electrical Properties of Polymer [1]}

The undoped polymer [1] is brownish powder, and its color changed from brownish to black when doped with iodine. Iodine is a convenient dopant in the laboratory and the doped amount in the polymer can be easily controlled. The polymer can be readily doped by iodine and the concentration of the doped iodine reached to about equimolar amount per polymer repeating unit within $90 \mathrm{~min}$. The electrical conductivity of the polymer is about $10^{-13} \mathrm{~S} \mathrm{~cm}^{-1}$ under the undoped condition, but increases up to $10^{-6} \mathrm{~S} \mathrm{~cm}^{-1}$ when it is exposed to the vapor of iodine at reduced

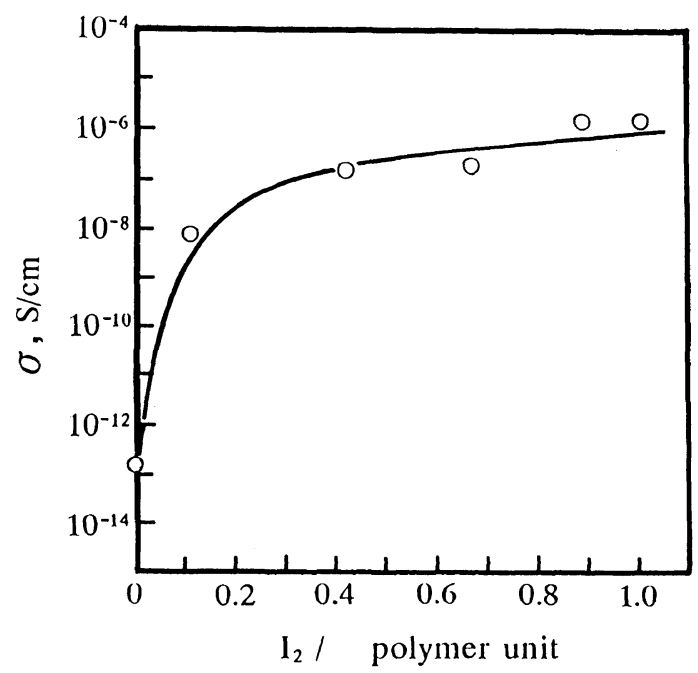

Figure 5. Conductivity of polymer (Table I, No. 3) at $25^{\circ} \mathrm{C}$ under nitrogen atmosphere: $\mathrm{I}_{2}$ doped at $40^{\circ} \mathrm{C}$, $0.5 \mathrm{mmHg}$. 


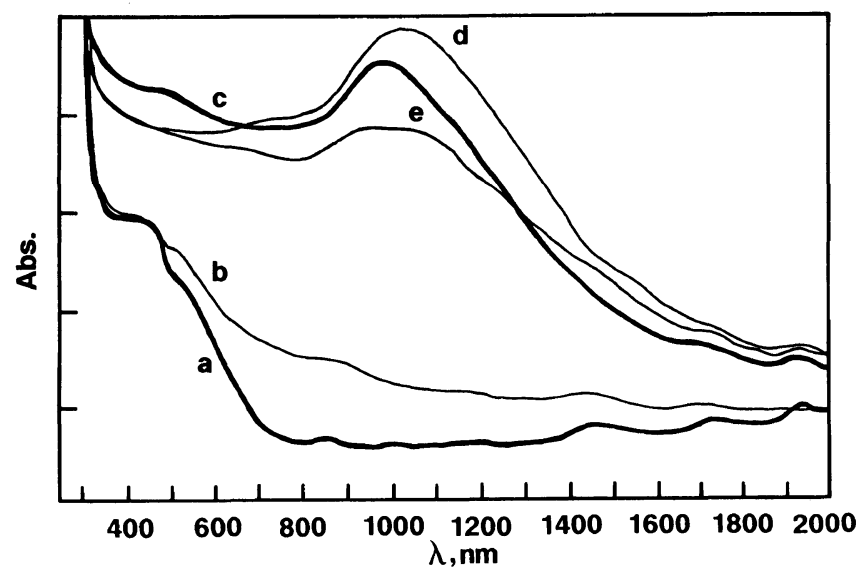

Figure 6. Diffuse reflectance spectra of DEA-BDT polymers (Table I, No. 3) with various $I_{2}$ doping levels. Doping level, $\mathrm{I}_{2}$ /polymer unit: a), $\left.\left.\left.\left.0 ; \mathrm{b}\right), 0.11 ; \mathrm{c}\right), 0.42 ; \mathrm{d}\right), 0.67 ; \mathrm{e}\right), 0.89$.

pressure. The relation between the electrical conductivity and the iodine concentration in the polymer is shown in Figure 5.

The lower conductivity of the polymer [1] than that of the polymer composed of 1,4diethynylbenzene and BDT seems to be caused by the selection of unsuitable dopants or by the lack of the stereo-structural regularity of the polymer [1] than the polymer composed of 1,4-diethynylbenzene and BDT, due to the steric hindrance of the large anthracene unit in the polymer backbone. The electrical conductivity would be improved, if the polymer is composed of the similar monomers having the same aromatic ring, so that the polymer chain easily forms a pile structure.

Figure 6 shows the diffuse reflectance spectra of the undoped and the iodine doped polymers. From these, it can be seen that the undoped polymer has a strong absorption at $428 \mathrm{~nm}$, and tailing up to $700 \mathrm{~nm}$. Also, there appear small peaks at $850,1435,1705$, and 1925 $\mathrm{nm}$. With the increase of the concentration of the doped iodine, the absorption intensity at $428 \mathrm{~nm}$ becomes weaker, however, the peak at $850 \mathrm{~nm}$ gradually becomes stronger accompanying with a little red shift similar to the case of polypyrrole. ${ }^{15}$ It is suggested that the strong interaction between vinylene anthra- cene units in the polymer backbone and iodine would form a kind of complex which is so-called a polaron or bipolaron. Though the existence of these kinds of complexes in the polymer backbone will have to be confirmed in a further study, it is understood that the formation of these charged complexes along the polymer backbone is responsible for the enhanced electrical conductivity.

\section{CONCLUSIONS}

A novel monomer of 9,10-diethynylanthracene was prepared from 9,10-dibromoanthracene by way of 9,10-bis(trimethylsilylethynyl)anthracene. A conjugated polymer of 9,10diethynylanthracene with 1,4-benzenedithiol was for the first time synthesized in organic solvents such as benzene and THF at a proper temperature by UV irradiation or using radical initiators. The molecular weight $\left(\bar{M}_{n}\right)$ of the THF insoluble polymer is about 80000 and the THF soluble one about 6000 , which is quite different from that of the polymer obtained in a benzene solution. From the sulfur content and IR spectrum of the insoluble polymer, it is considered that the obtained polymer [1] has the alternating structure composed of 9,10-diethynylanthracene and 1,4- 
benzenedithiol units. The polymer was very rapidly doped in the vapor of iodine under reduced pressure. The color of the polymer changed from brownish to black on doping of iodine. The electrical conductivity of undoped polymer is about $10^{-13} \mathrm{Scm}^{-1}$, but increases up to $10^{-6} \mathrm{Scm}^{-1}$ by doping with iodine. From the diffuse reflectance spectra of the doped polymer, it is suggested that a strong interaction between the vinylene anthracene unit in the polymer backbone and iodine induced the formation of a polaron or bipolaron along the polymer backbone.

\section{REFERENCES}

1. E. Kobayashi, T. Ohashi, and J. Furukawa, Makromol. Chem., 187, 2525 (1986).

2. E. Kobayashi, T. Ohashi, and J. Furukawa, J. Polym. Sci., Part A, Polym. Chem. Ed., 25, 2077 (1987).

3. E. Kobayashi, T. Ohashi, and J. Furukawa, Polym. J., 21, 111 (1989).
4. E. Kobayashi, T. Ohashi, T. Yagi, and J. Furukawa, Rep. Jpn. Synthetic Fiber Institute, 44, 95 (1987).

5. E. Kobayashi, T. Kaneda, T. Ohashi, and J. Furukawa, Polym. Prepr. Jpn., 37, 264 (1988).

6. E. Kobayashi, T. Ohashi, and J. Furukawa, Polym. Prepr. Jpn., 37, 263 (1988).

7. E. Kobayashi, T. Miyaji, T. Ohashi, and J. Furukawa, Polym. Prepr. Jpn., 36, 291 (1987).

8. E. Kobayashi, Y. Ishizuka, Y. Kouchaku, and J. Furukawa, Kobunshi Ronbunshu, 46, 203 (1989).

9. O. Nuyken and F. Siebzehnrübl, Polym. Bull., 19, 371 (1987).

10. Synthesized according to the literature, "Organic Syntheses," Coll. Vol. 1, John Wiley \& Sons, Inc., New York, N. Y., 1941, p 207.

11. Synthesized according to the literature, H. Itatani and J. C. Bailar, Jr., J. Am. Oil Chem. Soc., 44, 147 (1967).

12. V. C. Pareke, J. Indian Chem. Soc., 11, 95 (1934).

13. E. Kobayashi, T. Ohashi, and J. Furukawa, Rep. Jpn. Synthetic Fiber Institute, 43, 95 (1986).

14. J. R. Allen and L. Henning, "Encyclopedia of Electrochemistry of the Elements," Organic Section, Vol. XI, Marcel Dekker Inc., New York, N. Y., 1978, p 48, 117.

15. J. L. Bredas, J. C. Scott, K. Yakushi, and G. B. Street, Phys. Rev. B, 30, 1023 (1984). 